\title{
Largest Family Without a Pair of Posets on Consecutive Levels of the Boolean Lattice
}

\author{
Gyula O. H. Katona ${ }^{1}$ (D) . Jimeng Xiao ${ }^{2,3}$
}

Received: 20 March 2020 / Accepted: 15 February 2021 / Published online: 12 March 2021

(C) The Author(s) 2021

\begin{abstract}
Suppose $k \geq 2$ is an integer. Let $Y_{k}$ be the poset with elements $x_{1}, x_{2}, y_{1}, y_{2}, \ldots, y_{k-1}$ such that $y_{1}<y_{2}<\cdots<y_{k-1}<x_{1}, x_{2}$ and let $Y_{k}^{\prime}$ be the same poset but all relations reversed. We say that a family of subsets of [n] contains a copy of $Y_{k}$ on consecutive levels if it contains $k+1$ subsets $F_{1}, F_{2}, G_{1}, G_{2}, \ldots, G_{k-1}$ such that $G_{1} \subset G_{2} \subset \cdots \subset G_{k-1} \subset$ $F_{1}, F_{2}$ and $\left|F_{1}\right|=\left|F_{2}\right|=\left|G_{k-1}\right|+1=\left|G_{k-2}\right|+2=\cdots=\left|G_{1}\right|+k-1$. If both $Y_{k}$ and $Y_{k}^{\prime}$ on consecutive levels are forbidden, the size of the largest such family is denoted by $\mathrm{La}_{\mathrm{c}}\left(n, Y_{k}, Y_{k}^{\prime}\right)$. In this paper, we will determine the exact value of $\operatorname{La}_{\mathrm{c}}\left(n, Y_{k}, Y_{k}^{\prime}\right)$.
\end{abstract}

Keywords Forbidden subposets $\cdot$ Extremal set theory $\cdot$ Double counting

\section{Introduction}

Given two partially ordered sets (posets) $P$ and $Q$, we say that $P$ is a subposet of $Q$ if there exists an injection $\phi: P \rightarrow Q$ such that $x \leq_{P} \quad y$ implies $\phi(x) \leq Q \quad \phi(y)$. Viewing collections of sets as posets under the inclusion relation, we have the following extremal functions, first introduced by Katona and Tarján [9]. For any collection of finite posets $\mathcal{P}$, let $\mathrm{La}(n, \mathcal{P})$ be the maximum size of a family of subsets of $[n]=\{1,2, \ldots, n\}$ which does not contain any $P \in \mathcal{P}$ as a subposet.

This type of problems was first studied by Sperner [14].

Theorem 1.1 (Sperner [14]) Let $\mathcal{F}$ be a family of subsets of $[n]$ without inclusion relation between any two of the subsets. Then

$$
|\mathcal{F}| \leq\left(\begin{array}{c}
n \\
\left\lfloor\frac{n}{2}\right\rfloor
\end{array}\right) .
$$

Gyula O. H. Katona

katona.gyula.oh@renyi.hu

Alfréd Rényi Institute of Mathematics, Budapest, Hungary

2 School of Mathematics and Statistics, Northwestern Polytechnical University, Xi'an, People's Republic of China

3 Xi' an-Budapest Joint Research Center for Combinatorics, Northwestern Polytechnical University, Xi' an, People's Republic of China 
A chain of length $k$ is a poset with elements $x_{1}, x_{2}, \ldots, x_{k}$ such that $x_{1}<x_{2}<\cdots<x_{k}$. Let $\sum(n, k)$ be the sum of the $k$ largest binomial coefficients of the form $\left(\begin{array}{l}n \\ i\end{array}\right)$. Then Sperner's theorem can be extended as follows:

Theorem 1.2 (Erdős [3]) Let $\mathcal{F}$ be a family of subsets of [n] without a chain of length $k$. Then

$$
|\mathcal{F}| \leq \sum(n, k-1)
$$

Before stating the next result, we need the following notation. Let $2 \leq k \leq n$ and $0 \leq r \leq n$ be two integers. The following lacunary sum of binomial coefficients was first introduced by Ramus [13] in 1834 .

$$
S(n, k, r)=\sum_{\substack{i=0 \\
i \equiv r \bmod k}}^{n}\left(\begin{array}{l}
n \\
i
\end{array}\right) .
$$

Clearly, if $r_{1} \equiv r_{2}(\bmod k)$, then $S\left(n, k, r_{1}\right)=S\left(n, k, r_{2}\right)$. So there are $k$ distinct such sums $S(n, k, 0), S(n, k, 1), \ldots, S(n, k, k-1)$.

The first author [8] published the next two theorems which are analogs of the two theorems above.

Theorem 1.3 (Katona [8]) Let $\mathcal{F}$ be a family of subsets of $[n]$ such that no two of the subsets $F_{i}, F_{j}$ satisfy $F_{i} \subset F_{j}$ and $\left|F_{j}\right|-\left|F_{i}\right|<k$. Then

$$
|\mathcal{F}| \leq \max \{S(n, k, r) \mid r \in\{0, \ldots, k-1\}\} .
$$

Theorem 1.4 (Katona [8]) Let $h \leq k$ be an integer, and let $\mathcal{F}$ be a family of subsets of [ $n]$ such that no $h+1$ of the subsets $F_{i_{1}}, \ldots, F_{i_{h+1}}$ satisfy $F_{i_{1}} \subset \cdots \subset F_{i_{h+1}}$ and $\left|F_{i_{h+1}}\right|-$ $\left|F_{i_{1}}\right|<k$. Then

$|\mathcal{F}| \leq \max \left\{S\left(n, k, r_{1}\right)+S\left(n, k, r_{2}\right)+\cdots+S\left(n, k, r_{h}\right) \mid r_{1} \neq r_{2} \neq \cdots \neq r_{h} \in\{0, \ldots, k-1\}\right\}$.

Another type of generalizations of Sperner Theorem is to determine the largest size of a family of subsets of $[n]$ without a copy of poset $Y_{k}$ and $Y_{k}^{\prime}$ defined below. Let $Y_{k}$ be the poset with elements $x_{1}, x_{2}, y_{1}, y_{2}, \ldots, y_{k-1}$ such that $y_{1}<y_{2}<\cdots<y_{k-1}<x_{1}, x_{2}$, and let $Y_{k}^{\prime}$ be the same poset but all relations reversed. Katona and Tarján [9] gave the following result for $k=2$. (In their paper, posets $Y_{2}$ and $Y_{2}^{\prime}$ are denoted by $\mathrm{V}$ (the cherry poset) and $\Lambda$ (the fork poset) respectively.)

Theorem 1.5 (Katona and Tarján [9])

$$
\mathrm{La}\left(t n, Y_{2}, Y_{2}^{\prime}\right)=2\left(\begin{array}{c}
n-1 \\
\left\lfloor\frac{n-1}{2}\right\rfloor
\end{array}\right) .
$$

De Bonis, Katona and Swanepoel [2] studied the case $k=3$. We remark that they actually proved a result for the so-called butterfly poset, but their proof implies the following theorem.

Theorem 1.6 (De Bonis, Katona and Swanepoel [2])

$$
\mathrm{La}\left(n, Y_{3}, Y_{3}^{\prime}\right)=\sum(n, 2) .
$$


For the case $k \geq 4$, Methuku and Tompkins [12] got the next theorem.

Theorem 1.7 (Methuku and Tompkins [12]) For $n \geq k \geq 4$,

$$
\mathrm{La}\left(n, Y_{k}, Y_{k}^{\prime}\right)=\sum(n, k-1) .
$$

For other results related to the La function, see $[1,5-7,11,15]$. Now, we consider the following problem, which is a combination of the two types of problems above. We say that a family of subsets of $[n]$ contains a copy of $Y_{k}$ on consecutive levels if it contains $k+1$ subsets $F_{1}, F_{2}, G_{1}, G_{2}, \ldots, G_{k-1}$ such that $G_{1} \subset G_{2} \subset \cdots \subset G_{k-1} \subset F_{1}, F_{2}$ and $\left|F_{1}\right|=\left|F_{2}\right|=\left|G_{k-1}\right|+1=\left|G_{k-2}\right|+2=\cdots=\left|G_{1}\right|+k-1$. We denote by $\operatorname{La}_{\mathrm{c}}\left(n, Y_{k}, Y_{k}^{\prime}\right)$ the largest size of a family of subsets of [n] containing neither $Y_{k}$ nor $Y_{k}^{\prime}$ on consecutive levels. We note that this problem is mentioned in [4] in the language of the oriented hypercube. The authors in [4] gave an asymptotic formula of the size of largest family without tree posets in consecutive levels. For exact result, they proved

$$
\operatorname{La}_{\mathrm{c}}\left(n, Y_{2}, Y_{2}^{\prime}\right)=2^{n-1} .
$$

Let $m=\lceil(n-k) / 2\rceil$ in the rest of this paper. For $k \geq 3$, we have the following theorem.

Theorem 1.8 Let $n \geq k \geq 3$, then

$$
\operatorname{La}_{\mathrm{c}}\left(n, Y_{k}, Y_{k}^{\prime}\right)=2^{n}-S(n, k, m) .
$$

Remark 1.9 (I) Loehr and Michael [10] showed that

$$
S(n, k, m)=\min _{r: 0 \leq r \leq k-1} S(n, k, r) .
$$

So our result implies that the trivial construction is the best. Here, a trivial construction consists of all subsets except the ones with size $s \equiv m(\bmod k)$.

(II) If $n=k \geq 3$, then $m=0$ and $S(n, k, m)=\left(\begin{array}{l}n \\ 0\end{array}\right)+\left(\begin{array}{l}n \\ n\end{array}\right)=2$. In this case, Theorem 1.8 is trivial, since every family $\mathcal{F}$ with size $2^{n}-1$ contains a copy of $Y_{k}$ or $Y_{k}^{\prime}$ on consecutive levels.

The rest of the paper is organized as follows. In the next section, we present some preliminary results. In Section 3, we will prove our main theorem (Theorem 2.1), which implies Theorem 1.8.

\section{Preliminary Results}

A cyclic permutation $\sigma$ of $[n]$ is a cyclic ordering $a_{1}, a_{2}, \ldots, a_{n}, a_{1}$, where $a_{i} \in[n]$ for $i=$ $1,2, \ldots, n$. Let $\mathcal{F}$ be a family of subsets of $[n]$ containing neither $Y_{k}$ nor $Y_{k}^{\prime}$ on consecutive levels. We say a set $F \in \sigma$ if $F$ is an interval along the cyclic permutation $\sigma$.

Now, we double count the sum $S$ of $\phi_{F}$ over all cyclic permutations $\sigma$ and $F \in \mathcal{F}$ such that $F \in \sigma$, where

$$
\phi_{F}= \begin{cases}\left(\begin{array}{l}
n \\
|F|
\end{array}\right), & \text { if } F \neq \emptyset \text { and } F \neq[n] ; \\
n, & \text { if } F=\emptyset \text { or } F=[n] .\end{cases}
$$


For any $F \in(\mathcal{F} \backslash\{\emptyset,[n]\})$, we have $|F| !(n-|F|)$ ! cyclic permutations $\sigma$ satisfying $F \in \sigma$. If $\emptyset \in \mathcal{F}$ or $[n] \in \mathcal{F}$, all cyclic permutations satisfy the above condition, and the number of cyclic permutations is $(n-1)$ !. So

$$
\begin{aligned}
S & =|\{\emptyset,[n]\} \cap \mathcal{F}| \cdot n \cdot(n-1) !+\sum_{F \in(\mathcal{F} \backslash\{\emptyset,[n]\})}|F| !(n-|F|) !\left(\begin{array}{l}
n \\
|F|
\end{array}\right) \\
& =\left(|\{\emptyset,[n]\} \cap \mathcal{F}|+\sum_{F \in(\mathcal{F} \backslash\{\emptyset,[n]\})} 1\right) \cdot n !=|\mathcal{F}| \cdot n !
\end{aligned}
$$

On the other hand, for any cyclic permutation $\sigma$, let $\mathcal{I}(n)^{\sigma}$ be the family of intervals along $\sigma$. The $i^{\text {th }}$ level of $\mathcal{I}(n)^{\sigma}$ is the collection of its elements of size $i$. (See Fig. 1 for an example of $\mathcal{I}(6)^{\sigma}$, where $\sigma=1,2,3,4,5,6,1$.) For $0 \leq i \leq n$, let $x_{i}$ be the number of subsets in the $i^{\text {th }}$ level of $\mathcal{F} \cap \mathcal{I}(n)^{\sigma}$. Then

$$
S=\sum_{\sigma}\left(|\{\emptyset,[n]\} \cap \mathcal{F}| \cdot n+\sum_{i=1}^{n-1}\left(\begin{array}{l}
n \\
i
\end{array}\right) x_{i}\right) .
$$

Now, we need the following theorem to give an upper bound on $|\{\emptyset,[n]\} \cap \mathcal{F}| \cdot n+$ $\sum_{i=1}^{n-1}\left(\begin{array}{l}n \\ i\end{array}\right) x_{i}$ for every cyclic permutation $\sigma$.

Theorem 2.1 Let $n>k \geq 3$ and $\mathcal{F}$ be a family of subsets of $[n]$ containing neither $Y_{k}$ nor $Y_{k}^{\prime}$ on consecutive levels. Then for every cyclic permutation $\sigma$, we have

$$
|\{\emptyset,[n]\} \cap \mathcal{F}| \cdot n+\sum_{i=1}^{n-1}\left(\begin{array}{l}
n \\
i
\end{array}\right) x_{i} \leq n \cdot\left(2^{n}-S(n, k, m)\right)+n-1 .
$$

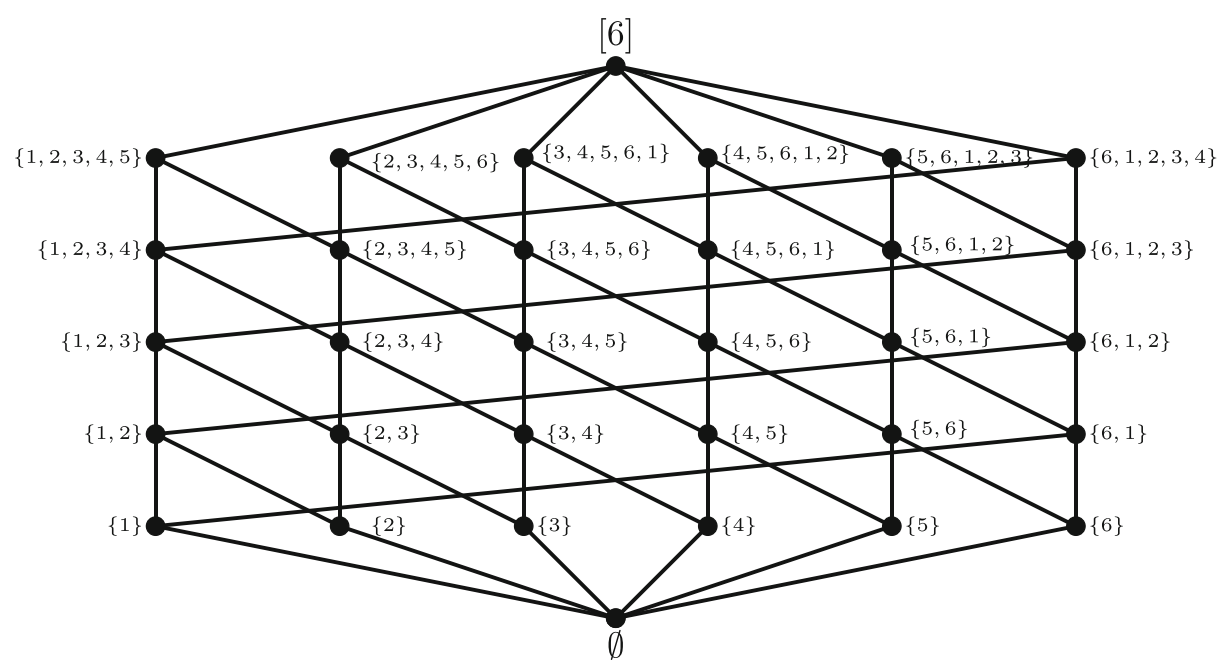

Fig. $1 \mathcal{I}(n)^{\sigma}$ for $n=6$ and $\sigma=1,2,3,4,5,6,1$ is shown by bold vertices, and two vertices from consecutive levels are connected by an edge if they have inclusion relation 
Supposing that we know Theorem 2.1, then

$$
S=|\mathcal{F}| \cdot n ! \leq \sum_{\sigma}\left(n \cdot\left(2^{n}-S(n, k, m)\right)+n-1\right)=(n-1) ! \cdot\left(n \cdot\left(2^{n}-S(n, k, m)\right)+n-1\right) .
$$

Since $|\mathcal{F}|$ is an integer, we have

$$
|\mathcal{F}| \leq\left\lfloor\frac{n \cdot\left(2^{n}-S(n, k, m)\right)+n-1}{n}\right\rfloor=2^{n}-S(n, k, m),
$$

as desired. So by (II) of Remark 1.9, it is sufficient to prove Theorem 2.1. The following two lemmas are needed to give constraints of $x_{0}, x_{1}, \ldots, x_{n}$.

Lemma 2.2 If $0 \leq i \leq n-k+1$, then for all $\sigma$,

$$
x_{i}+x_{i+1}+\cdots+x_{i+k-1} \leq(k-1) n .
$$

Lemma 2.3 For all $\sigma$, if $x_{0}=1$ and $x_{1}+x_{2}+\cdots+x_{k}=(k-1) n$, then

$$
x_{0}+x_{1}+\cdots+x_{k-1} \leq(k-1) n-\lfloor n / 2\rfloor .
$$

For all $\sigma$, if $x_{n}=1$ and $x_{n-k}+x_{n-k+1}+\cdots+x_{n-1}=(k-1) n$, then

$$
x_{n-k+1}+x_{n-k+2}+\cdots+x_{n} \leq(k-1) n-\lfloor n / 2\rfloor \text {. }
$$

Before starting the proofs of the two lemmas above, we introduce some notations. Let $\sigma=a_{1}, a_{2}, \ldots, a_{n}, a_{1}$, where $a_{j} \in[n]$ for $1 \leq j \leq n$. In the rest of this paper, we view $\mathcal{I}(n)^{\sigma}$ (a subfamily of $\mathcal{I}(n)^{\sigma}$ ) as a graph. The vertex set of this graph is $\mathcal{I}(n)^{\sigma}$ (the subfamily of $\left.\mathcal{I}(n)^{\sigma}\right)$, and two vertices are connected by an edge if they are from consecutive levels and they have inclusion relation (see Fig. 2). We denote by $I_{t}^{s}$ the vertex $\left\{a_{t+1}, a_{t+2}, \ldots, a_{t+s}\right\}$ (with addition taken modulo $n$ ) of $\mathcal{I}(n)^{\sigma}$. Clearly, $\left|I_{t}^{s}\right|=s, I_{t}^{s-1}, I_{t+1}^{s-1} \subset I_{t}^{s}$ for $2 \leq s \leq$ $n-1$, and $I_{t}^{s} \subset I_{t-1}^{s+1}, I_{t}^{s+1}$ for $1 \leq s \leq n-2$ (see Fig. 2).

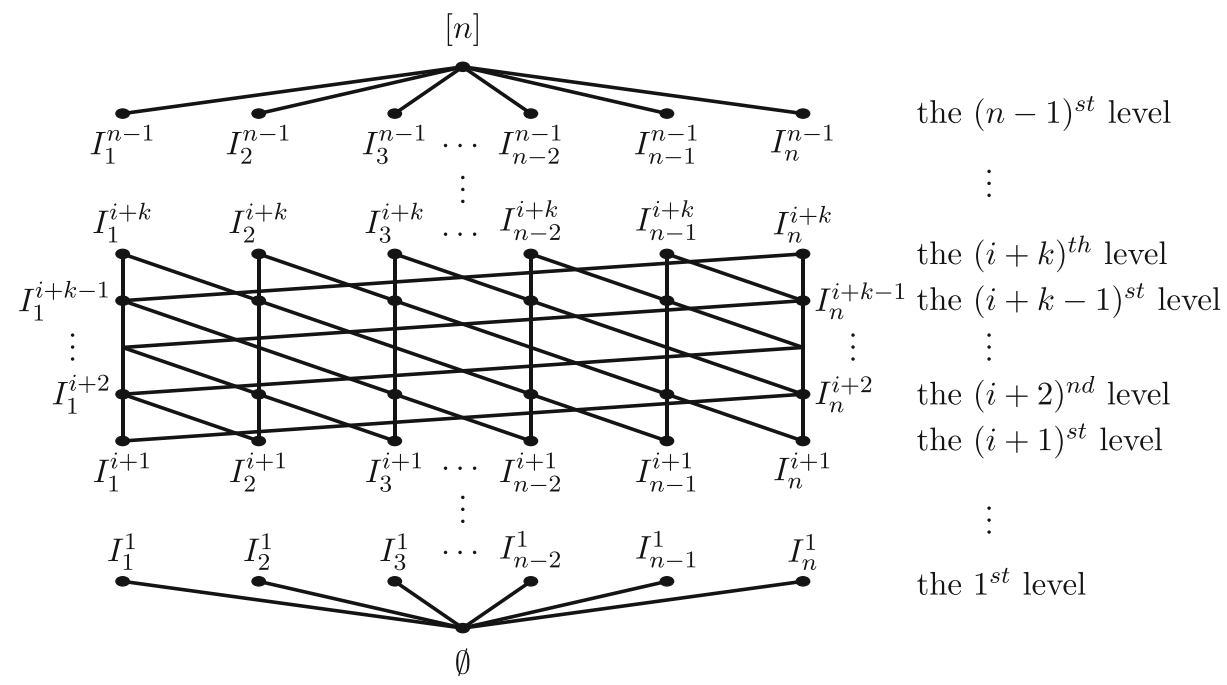

Fig. 2 Vertices $I_{t}^{s}$ of $\mathcal{I}(n)^{\sigma}$ 
In order to prove the two kinds of constraints of $k$ consecutive $x_{i}$ 's above, we consider some typical structures in $k$ consecutive levels of $\mathcal{I}(n)^{\sigma}$. If the $k$ levels are the levels from $0^{t h}$ to $(k-1)^{s t}$, then every $Y_{k}$ on these levels must contain $\emptyset$. In this case, we consider a special kind of $Y_{k}$, which we denote by

$$
Y_{k}(j)=\left\{\emptyset, I_{j}^{1}, I_{j}^{2}, \ldots, I_{j}^{k-2}, I_{j}^{k-1}, I_{j-1}^{k-1}\right\}
$$

where $1 \leq j \leq n$. (See Fig. 3 for an example of $Y_{k}(1)$.) If the $k$ levels are the levels from $(i+1)^{s t}$ to $(i+k)^{t h}$ (the middle part of Fig. 2), then we introduce a new kind of structure on $k$ consecutive levels. A family of $k+2$ subsets is called $X_{k}$ if it is

$\left\{I_{t+1}^{i+1}, I_{t}^{i+1}, I_{t}^{i+2}, I_{t}^{i+3}, \ldots, I_{t}^{i+k}, I_{t-1}^{i+k}\right\}$ or $\left\{I_{t-1}^{i+1}, I_{t}^{i+1}, I_{t-1}^{i+2}, I_{t-2}^{i+3}, \ldots, I_{t-k+1}^{i+k}, I_{t-k+2}^{i+k}\right\}$,

where $1 \leq t \leq n$. (See Fig. 3 for two types of examples of $t=2$ and $t=k$ respectively.) In each $X_{k}$, we call the 3 elements in the $(i+k)^{t h}$ level and the $(i+k-1)^{s t}$ level a cherry, and the 3 elements in the $(i+1)^{s t}$ level and the $(i+2)^{n d}$ level a fork.

Remark 2.4 (I) The vertices of the $n Y_{k}$ 's $\left(Y_{k}(1), Y_{k}(2), \ldots, Y_{k}(n)\right)$ cover the vertices of the levels of $\mathcal{I}(n)^{\sigma}$ from $1^{\text {st }}$ to $(k-2)^{\text {nd }}$ once.

(II) For $k \geq 3$ and $0 \leq i \leq n-k-1$, the edges of the $2 n X_{k}$ 's cover the edges within the $(i+1)^{s t}$ level and the $(i+2)^{n d}$ level of $\mathcal{I}(n)^{\sigma}$ twice, the edges within the levels of $\mathcal{I}(n)^{\sigma}$ from $(i+2)^{n d}$ to $(i+k-1)^{s t}$ once, and the edges within the $(i+k-1)^{s t}$ level and the $(i+k)^{t h}$ level of $\mathcal{I}(n)^{\sigma}$ twice.

Now, we are ready to start the proofs of Lemmas 2.2 and 2.3. We note that a vertex $I$ of $\mathcal{I}(n)^{\sigma}$ is a subset of $[n]$. So in the two proofs, we write $I \in \mathcal{F}$ if the subset $I$ is an element of the family $\mathcal{F}$.

Proof of Lemma 2.2 First, we show $x_{0}+x_{1}+\cdots+x_{k-1} \leq(k-1) n$. Since $x_{0} \leq 1$ and $x_{i} \leq n$ for $1 \leq i \leq k-1$, we have $x_{0}+x_{1}+\cdots+x_{k-1} \leq(k-1) n+1$. If $x_{0}+x_{1}+\cdots+x_{k-1}=(k-1) n+1$, every subset of the levels from $0^{\text {th }}$ to $(k-1)^{s t}$ is in
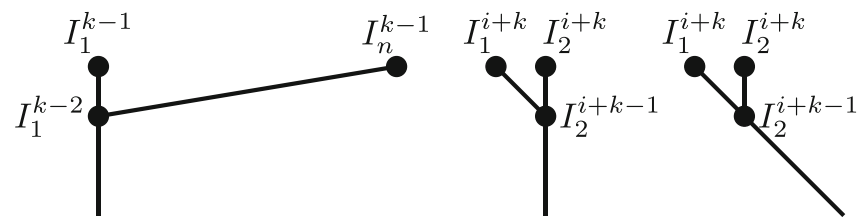

cherry
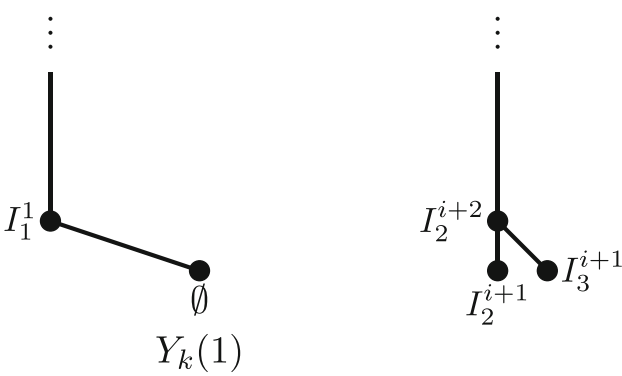

$X_{k}$

Fig. 3 Examples of structures on $k$ consecutive levels 
$\mathcal{F}$, then one can easily find a copy of $Y_{k}$ on consecutive levels. (See $Y_{k}(1)$ for an example in Fig. 3.) Similarly, we have $x_{n-k+1}+x_{n-k+2}+\cdots+x_{n} \leq(k-1) n$.

Now, we prove that

$$
x_{i+1}+x_{i+2}+\cdots+x_{i+k} \leq(k-1) n
$$

for $0 \leq i \leq n-k-1$. Recall that there is an edge between two vertices $I, I^{\prime}$ if and only if $I^{\prime} \subset I$ and $|I|-\left|I^{\prime}\right|=1$. Then, we double count the sum $T$ of the weight function $\psi(e)$ over all edges $e=\left\{I, I^{\prime}\right\}$ in the $2 n X_{k}$ 's, where

$$
\psi(e)= \begin{cases}0, & \text { if } I, I^{\prime} \in \mathcal{F} ; \\ 0, & \text { if } I, I^{\prime} \notin \mathcal{F} \\ 0, & I \in \mathcal{F}, I^{\prime} \notin \mathcal{F} \text { and }|I|=i+k \\ 0, & I \notin \mathcal{F}, I^{\prime} \in \mathcal{F} \text { and }|I|=i+2 \\ 1, & \text { otherwise. }\end{cases}
$$

On the one hand, recall that every vertex in the $s^{\text {th }}$ level of $\mathcal{I}(n)^{\sigma}$ have two neighbors in the $(s+1)^{s t}$ level and two neighbors in the $(s-1)^{s t}$ level, and note that only the case when one of the two vertices in the edge is not in $\mathcal{F}$ counts nonzero. Then by (II) of Remark 2.4,

$$
\begin{aligned}
T= & \left(2 \cdot \sum_{I \notin \mathcal{F},|I|=i+k} \psi(e)+\sum_{I \notin \mathcal{F},|I| \neq i+k} \psi(e)\right)+\left(2 \cdot \sum_{I^{\prime} \notin \mathcal{F},\left|I^{\prime}\right|=i+1} \psi(e)+\sum_{I^{\prime} \notin \mathcal{F},\left|I^{\prime}\right| \neq i+1} \psi(e)\right) \\
& \leq\left(2 \cdot 2\left(n-x_{i+k}\right)+1 \cdot \sum_{j=i+3}^{i+k-1} 2\left(n-x_{j}\right)\right)+\left(2 \cdot 2\left(n-x_{i+1}\right)+1 \cdot \sum_{l=i+2}^{i+k-2} 2\left(n-x_{l}\right)\right) \\
= & 4\left(\sum_{h=i+1}^{i+k}\left(n-x_{h}\right)\right)-2\left(n-x_{i+2}\right)-2\left(n-x_{i+k-1}\right) .
\end{aligned}
$$

On the other hand, we will prove below the claim that in each $X_{k}$, the sum of the weight function over its edges is at least $\left|\left\{I \in \mathcal{F} \cap X_{k}|| I \mid=i+2\right\}\right|+\mid\left\{I \in \mathcal{F} \cap X_{k}|| I \mid=\right.$ $i+k-1\} \mid$. Then if we sum over all $2 n X_{k}$ 's, $T$ is at least $2\left(x_{i+2}+x_{i+k-1}\right)$.

Now, we divide the cherries of all $2 n X_{k}$ 's into 4 types (see Fig. 4), according to whether its 3 elements are in $\mathcal{F}$ or not. We call a cherry Type 1 ( 2 or 3 ) if the middle element and both (one or none) of its neighbors are in $\mathcal{F}$. The rest of the cases are Type 4, namely that the middle element is not in $\mathcal{F}$. Note that in Type 4, we do not distinguish the cases if two neighbors of the middle element are in $\mathcal{F}$ or not. Similarly, we divide all forks into Types 5, 6, 7 and 8 (see Fig. 4).

To prove the claim, we distinguish several cases by the types defined above. Note that in each $X_{k}$, in order to avoid copies of $Y_{k}$ and $Y_{k}^{\prime}$ in $\mathcal{F}$, either at least 2 of the 4 vertices in the $(i+1)^{s t}$ level and the $(i+k)^{t h}$ level are not in $\mathcal{F}$, or at least one of the $k-2$ vertices in the levels from $(i+2)^{n d}$ to $(i+k-1)^{s t}$ is not in $\mathcal{F}$. If the cherry and the fork in some $X_{k}$ are Types 1 and 5 respectively, then we must have some vertex $I$ not in $\mathcal{F}$ in the chain connecting the cherry and the fork in this $X_{k}$. Then along this chain in both directions from $I$, take the first vertices in $\mathcal{F}$ respectively. Thus, we can find at least two edges with weight 1. Note that such vertices exist since in Types 1 and 5 , the vertices in the $(i+2)^{\text {nd }}$ level and the $(i+k-1)^{s t}$ level are in $\mathcal{F}$. The same argument works for the pairs Types 1 and 6 , and Types 2 and 5. If they are Types 1 and 7, then we already have two desired edges in the fork. If they are Types 1 and 8, then we find the first vertex in $\mathcal{F}$ along the chain from 


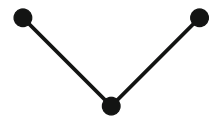

Type 1

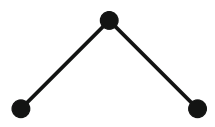

Type 5

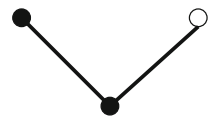

Type 2

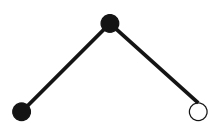

Type 6

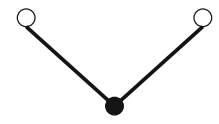

Type 3

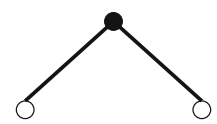

Type 7

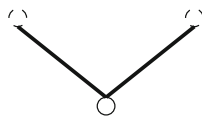

Type 4

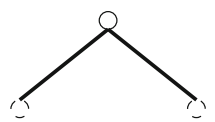

Type 8

Fig. 4 Types 1-8: the vertices $I$ in $\mathcal{F}$ (not in $\mathcal{F}$ or not clear) are denoted by solid (hollow or dashed) vertices, respectively

the fork to the cherry, this vertex and its neighbor below in the chain will give the edge we want. The same argument works for Types 4 and 5. In the rest cases, it is easy to find the desired edges in the cherry and the fork. (If $k=3$, Types 1 or 2 or 3 and 8 (5 or 6 or 7 and 4) cannot appear in any $X_{k}$, since $i+1=i+k-2$. If $k=3$ or 4, Types 1 and 5 ( 1 and 6 , or 2 and 5) cannot appear in any $X_{k}$, since they will form a $Y_{k}$ or a $Y_{k}^{\prime}$ in $\mathcal{F}$.)

Therefore, we have

$$
2\left(x_{i+2}+x_{i+k-1}\right) \leq 4\left(\sum_{h=i+1}^{i+k}\left(n-x_{h}\right)\right)-2\left(n-x_{i+2}\right)-2\left(n-x_{i+k-1}\right) .
$$

That is,

$$
x_{i+1}+x_{i+2}+\cdots+x_{i+k} \leq(k-1) n,
$$

as required.

Proof of Lemma 2.3 By symmetry, it is enough to prove the first part of the lemma. That is, we need to prove

$$
x_{0}+x_{1}+\cdots+x_{k-1} \leq(k-1) n-\lfloor n / 2\rfloor=(k-2) n+\lceil n / 2\rceil .
$$

We distinguish three cases to prove it.

Case $1 x_{k-1} \leq\lfloor n / 2\rfloor$ when $n$ is odd or $x_{k-1} \leq(n / 2)-1$ when $n$ is even.

Note that $x_{i} \leq n$ for $i=1,2, \ldots, k-2$, then by the assumption $x_{0}=1$, we have

$$
x_{0}+x_{1}+\cdots+x_{k-1} \leq 1+(k-2) n+\lfloor n / 2\rfloor \leq(k-2) n+\lceil n / 2\rceil,
$$

if $n$ is odd; and

$$
x_{0}+x_{1}+\cdots+x_{k-1} \leq 1+(k-2) n+(n / 2)-1 \leq(k-2) n+(n / 2),
$$

if $n$ is even.

Case $2 x_{k-1}=n / 2$ when $n$ is even.

Suppose that the result is not true. That is,

$$
x_{0}+x_{1}+\cdots+x_{k-1} \geq(k-2) n+(n / 2)+1 .
$$


Note that $x_{k-1}=n / 2$ in this case and $x_{i} \leq n$ for $i=1,2, \ldots, k-2$. Then by the assumption $x_{0}=1$, we have $x_{1}=x_{2}=\cdots=x_{k-2}=n$. Therefore, by the assumption $x_{1}+x_{2}+\cdots+x_{k}=(k-1) n$, we have $x_{k}=n / 2$. Since $x_{k-1}=n / 2$ and $x_{k}=n / 2$, we can find 2 vertices such that they are in the $k^{t h}$ level and the $(k-1)^{s t}$ level respectively, they are in $\mathcal{F}$, and they have inclusion relation. Then a copy of $Y_{k}^{\prime}$ on the levels from $1^{\text {st }}$ to $k^{\text {th }}$ can be found, a contradiction.

Case $3 x_{k-1} \geq\lfloor n / 2\rfloor+1$.

The number of pairs of vertices $\left(I_{j-1}^{k-1}, I_{j}^{k-1}\right)$ in $\mathcal{F}$ is at least $x_{k-1}-\left(n-x_{k-1}\right)=$ $2 x_{k-1}-n$ (see Fig. 5). To avoid a copy of $Y_{k}(j)$ in $\mathcal{F}$, at least one vertex of $Y_{k}(j)$ in the levels from $1^{s t}$ to $(k-2)^{n d}$ is not in $\mathcal{F}$. So by (I) of Remark 2.4, we have

$\sum_{i=0}^{k-1} x_{i} \leq 1+(k-2) n-\left(2 x_{k-1}-n\right)+x_{k-1}=1+(k-1) n-x_{k-1} \leq(k-1) n-\lfloor n / 2\rfloor$, since $x_{k-1} \geq\lfloor n / 2\rfloor+1$. This completes the proof of this case and the lemma.

Before starting the proof of Theorem 2.1, we need the following notations and lemmas for helping us to use the constraints above. Recall the definition of $S(n, k, r)$ and $m=$ $\lceil(n-k) / 2\rceil$. Now let $z$ be an integer such that $z \equiv r(\bmod k)$. Then we denote

$$
\begin{gathered}
S(n, k, r \mid z)=\sum_{\substack{i=0 \\
i \equiv r \bmod k \\
i \leq z}}^{n}\left(\begin{array}{l}
n \\
i
\end{array}\right) ; \\
w_{i}= \begin{cases}S(n, k, i \mid i)-S(n, k, i-1 \mid i-1), & \text { if } i \leq m ; \\
S(n, k, n-i-k+1 \mid n-i-k+1)-S(n, k, n-i-k \mid n-i-k), & \text { if } m+1 \leq i .\end{cases}
\end{gathered}
$$

Lemma 2.5 Let $t \leq n$ be an integer. We have the following equalities.

$$
\text { If } t<0, S(n, k, t \mid t)=0 \text {, and if } 0 \leq t \leq k-1, S(n, k, t \mid t)=\left(\begin{array}{c}
n \\
t
\end{array}\right) \text {. }
$$

Fig. 5 The cycle of the vertices in the $(k-1)^{s t}$ level of $\mathcal{I}(n)^{\sigma}$

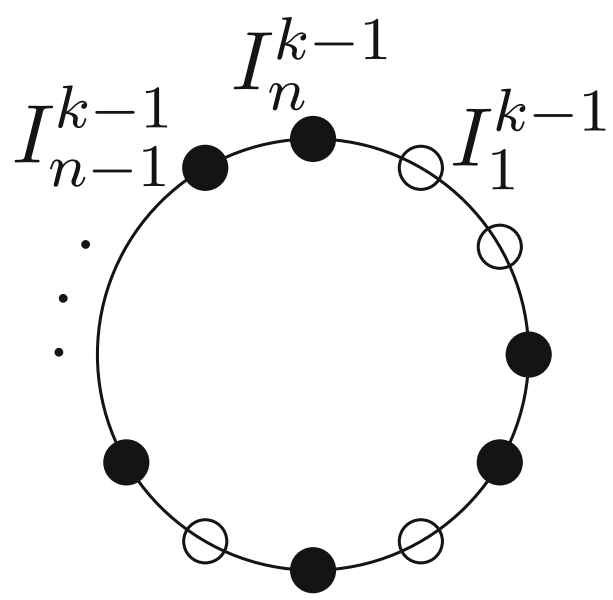


(II) If $0 \leq t \leq n, S(n, k, t \mid t)=S(n, k, t-k \mid t-k)+\left(\begin{array}{c}n \\ t\end{array}\right)$.

(III) If $0 \leq t \leq n, S(n, k, t \mid t)+S(n, k, n-k-t \mid n-k-t)=S(n, k, t)$.

(IV) If $0 \leq t \leq n, S(n, k, t-k \mid t-k)+S(n, k, n-k-t \mid n-k-t)=S(n, k, t)-\left(\begin{array}{c}n \\ t\end{array}\right)$.

Proof (I) follows from the definition of $S(n, k, t \mid t)$. (II) follows from (I) if $0 \leq t \leq k-1$, and the definition of $S(n, k, t \mid t)$ if $k \leq t \leq n$. (IV) follows from (II) and (III). Now, we distinguish two cases to prove (III).

If $t>n-k$, then we have $t+k>n$ and $n-k-t<0$. Hence, $S(n, k, t \mid t)=S(n, k, t)$ and $S(n, k, n-k-t \mid n-k-t)=0$ by (I), and this completes the proof of this case.

If $0 \leq t \leq n-k$, let $0 \leq r \leq k-1$ be the remainder of $n-k-t$ divided by $k$. We have

$$
\begin{aligned}
& S(n, k, t \mid t)+S(n, k, n-k-t \mid n-k-t) \\
= & S(n, k, t \mid t)+\left(\begin{array}{c}
n \\
r
\end{array}\right)+\left(\begin{array}{c}
n \\
r+k
\end{array}\right)+\cdots+\left(\begin{array}{c}
n \\
n-k-t
\end{array}\right) \\
= & S(n, k, t \mid t)+\left(\begin{array}{c}
n \\
n-r
\end{array}\right)+\left(\begin{array}{c}
n \\
n-r-k
\end{array}\right)+\cdots+\left(\begin{array}{c}
n \\
k+t
\end{array}\right) \\
= & S(n, k, t \mid t)+\left(\begin{array}{c}
n \\
t+k
\end{array}\right)+\cdots+\left(\begin{array}{c}
n \\
n-r-k
\end{array}\right)+\left(\begin{array}{c}
n \\
n-r
\end{array}\right)=S(n, k, t),
\end{aligned}
$$

since $r<k$.

\section{Lemma 2.6 Let $s$ be an integer.}

(I) $\quad w_{s}=0$ if $s<0$ or $s>n-k+1$, and $w_{s}>0$ if $0 \leq s \leq n-k+1$.

(II) If $0 \leq s \leq m$ or $m+k \leq s \leq n$, then

$$
\sum_{i=s-k+1}^{s} w_{i}=\left(\begin{array}{l}
n \\
s
\end{array}\right)
$$

(III) If $m<s<m+k$,

$$
\sum_{i=s-k+1}^{s} w_{i}=S(n, k, m)-S(n, k, s)+\left(\begin{array}{l}
n \\
s
\end{array}\right) .
$$

(IV) $\quad \sum_{i=0}^{n-k+1} w_{i}=S(n, k, m)$.

Proof (I) It follows from the definition of $w_{s}$ and (I) of Lemma 2.5.

(II) By (II) of Lemma 2.5, if $0 \leq s \leq m$, it follows from (I) that

$$
\sum_{i=s-k+1}^{s} w_{i}=\sum_{i=s-k+1}^{s}(S(n, k, i \mid i)-S(n, k, i-1 \mid i-1))=S(n, k, s \mid s)-S(n, k, s-k \mid s-k)=\left(\begin{array}{c}
n \\
s
\end{array}\right),
$$

and if $m+k \leq s \leq n$, we have $s-k+1 \geq m+1$, and so

$$
\begin{aligned}
\sum_{i=s-k+1}^{s} w_{i} & =\sum_{i=s-k+1}^{s}(S(n, k, n-k-i+1 \mid n-k-i+1)-S(n, k, n-k-i \mid n-k-i)) \\
& =S(n, k, n-s \mid n-s)-S(n, k, n-s-k \mid n-s-k)=\left(\begin{array}{c}
n \\
n-s
\end{array}\right)=\left(\begin{array}{c}
n \\
s
\end{array}\right) .
\end{aligned}
$$


(III) If $m<s<m+k$, then $s-k+1 \leq m$ and $s \geq m+1$. So by (III) and (IV) of Lemma 2.5, we have

$$
\begin{aligned}
\sum_{i=s-k+1}^{s} w_{i}= & \sum_{i=s-k+1}^{m}(S(n, k, i \mid i)-S(n, k, i-1 \mid i-1)) \\
& +\sum_{j=m+1}^{s}(S(n, k, n-k-j+1 \mid n-k-j+1)-S(n, k, n-k-j \mid n-k-j)) \\
= & S(n, k, m \mid m)-S(n, k, s-k \mid s-k)+S(n, k, n-k-m \mid n-k-m) \\
& -S(n, k, n-k-s \mid n-k-s) \\
= & (S(n, k, m \mid m)+S(n, k, n-k-m \mid n-k-m))-(S(n, k, s-k \mid s-k) \\
& +S(n, k, n-k-s \mid n-k-s)) \\
= & S(n, k, m)-\left(S(n, k, s)-\left(\begin{array}{c}
n \\
s
\end{array}\right)\right)=S(n, k, m)-S(n, k, s)+\left(\begin{array}{c}
n \\
s
\end{array}\right) .
\end{aligned}
$$

(IV)

$$
\sum_{i=0}^{n-k+1} w_{i}=\sum_{\substack{i=0 \\ i \equiv m \bmod k}}^{n}\left(\sum_{j=i-k+1}^{i} w_{j}\right)=S(n, k, m)
$$

by (I) and (II).

\section{Proof of Theorem 2.1}

First, we consider a linear programming problem: maximize $\sum_{i=0}^{n}\left(\begin{array}{l}n \\ i\end{array}\right) x_{i}$ subject to the constraints $x_{i}+x_{i+1}+\cdots+x_{i+k-1} \leq(k-1) n$ for $i=0,1 \ldots, n-k+1$ (Lemma 2.2), $0 \leq x_{0}, x_{n} \leq 1$, and $0 \leq x_{i} \leq n$ for all $i=1, \ldots, n-1$.

We assign the weight $w_{i}$ (see the definition and properties of $w_{i}$ in Section 2) to the constraint $x_{i}+x_{i+1}+\cdots+x_{i+k-1} \leq(k-1) n$ for every $0 \leq i \leq n-k+1$. By (I) of Lemma 2.6, $w_{i}>0$ for $0 \leq i \leq n-k+1$, so we have

$$
\sum_{i=0}^{n-k+1} w_{i}\left(x_{i}+x_{i+1}+\cdots+x_{i+k-1}\right) \leq(k-1) n \cdot \sum_{i=0}^{n-k+1} w_{i}
$$

Then by (I), (II) and (III) of Lemma 2.6, the LHS of (1) is equal to

$$
\sum_{i=0}^{n}\left(\left(\sum_{j=i-k+1}^{i} w_{j}\right) x_{i}\right)=\sum_{i=0}^{n}\left(\begin{array}{l}
n \\
i
\end{array}\right) x_{i}+\sum_{j=m+1}^{m+k-1}(S(n, k, m)-S(n, k, j)) x_{j} .
$$

On the other hand, by (IV) of Lemma 2.6, the RHS of (1) is equal to $(k-1) n \cdot S(n, k, m)$. Hence, by (1), we have

$$
\sum_{i=0}^{n}\left(\begin{array}{l}
n \\
i
\end{array}\right) x_{i}+\sum_{j=m+1}^{m+k-1}(S(n, k, m)-S(n, k, j)) x_{j} \leq(k-1) n \cdot S(n, k, m) .
$$


Note that $S(n, k, m)=\min _{r: 0 \leq r \leq k-1} S(n, k, r)$ by Remark 1.9, and $0 \leq x_{j} \leq n$ for $m+1 \leq j \leq m+k-1$. So we have

$$
\sum_{j=m+1}^{m+k-1}(S(n, k, j)-S(n, k, m)) x_{j} \leq \sum_{j=m+1}^{m+k-1}(S(n, k, j)-S(n, k, m)) n .
$$

Therefore, combining (2) and (3), we have

$$
\begin{aligned}
\sum_{i=0}^{n}\left(\begin{array}{l}
n \\
i
\end{array}\right) x_{i} & \leq(k-1) n \cdot S(n, k, m)+\sum_{j=m+1}^{m+k-1}(S(n, k, j)-S(n, k, m)) n \\
& =\sum_{j=m+1}^{m+k-1}(S(n, k, j)-S(n, k, m)+S(n, k, m)) n \\
& =n \cdot \sum_{j=m+1}^{m+k-1} S(n, k, j)=n \cdot\left(2^{n}-S(n, k, m)\right) .
\end{aligned}
$$

Now, we use (4) to prove Theorem 2.1. If $|\mathcal{F} \cap\{\emptyset,[n]\}|=0$, then the statement of the theorem follows from (4). Then if $|\mathcal{F} \cap\{\emptyset,[n]\}|=1$, we may suppose that $\emptyset \in \mathcal{F}$ and $[n] \notin \mathcal{F}$, and so $x_{0}=1$ and $x_{n}=0$. Thus, it follows that

$$
n \cdot x_{0}+\sum_{i=1}^{n-1}\left(\begin{array}{l}
n \\
i
\end{array}\right) x_{i}+n \cdot x_{n}=\sum_{i=0}^{n}\left(\begin{array}{l}
n \\
i
\end{array}\right) x_{i}+n-1 \leq n \cdot\left(2^{n}-S(n, k, m)\right)+n-1,
$$

as required.

If $\emptyset \in \mathcal{F}$ and $[n] \in \mathcal{F}$, we have $x_{0}=1$ and $x_{n}=1$. By Lemma 2.2, $x_{1}+x_{2}+\cdots+x_{k} \leq$ $(k-1) n$ and $x_{n-k}+x_{n-k+1}+\cdots+x_{n-1} \leq(k-1) n$. According to these two constraints, we distinguish two subcases.

If $x_{1}+x_{2}+\cdots+x_{k} \leq(k-1) n-1$ or $x_{n-k}+x_{n-k+1}+\cdots+x_{n-1} \leq(k-1) n-1$, we may assume $x_{1}+x_{2}+\cdots+x_{k} \leq(k-1) n-1$. Then in this case, (1) should be

$$
\sum_{i=0}^{n-k+1} w_{i}\left(x_{i}+x_{i+1}+\cdots+x_{i+k-1}\right) \leq\left((k-1) n \cdot \sum_{i=0}^{n-k+1} w_{i}\right)-w_{1} .
$$

By the assumption of Theorem 2.1,n>k, so $m \geq 1$ and $w_{1}=n-1$. Then (4) should be $\sum_{i=0}^{n}\left(\begin{array}{l}n \\ i\end{array}\right) x_{i} \leq n \cdot\left(2^{n}-S(n, k, m)\right)-(n-1)$, and so

$$
\begin{aligned}
& n\left(x_{0}+x_{n}\right)+\sum_{i=1}^{n-1}\left(\begin{array}{l}
n \\
i
\end{array}\right) x_{i}=(n-1)\left(x_{0}+x_{n}\right)+\sum_{i=0}^{n}\left(\begin{array}{l}
n \\
i
\end{array}\right) x_{i} \\
& \leq 2(n-1)+n \cdot\left(2^{n}-S(n, k, m)\right)-(n-1) \\
& =n \cdot\left(2^{n}-S(n, k, m)\right)+(n-1) .
\end{aligned}
$$

If $x_{1}+x_{2}+\cdots+x_{k}=(k-1) n$ and $x_{n-k}+x_{n-k+1}+\cdots+x_{n-1}=(k-1) n$, then by Lemma $2.3, x_{0}+x_{1}+\cdots+x_{k-1} \leq(k-1) n-\left\lfloor\frac{n}{2}\right\rfloor$ and $x_{n-k+1}+x_{n-k+2}+\cdots+x_{n} \leq(k-1) n-\left\lfloor\frac{n}{2}\right\rfloor$. Similarly, one can modify (1) and (4) to show that

$$
\begin{aligned}
n \cdot\left(x_{0}+x_{n}\right)+\sum_{i=1}^{n-1}\left(\begin{array}{l}
n \\
i
\end{array}\right) x_{i} & \leq 2(n-1)+n \cdot\left(2^{n}-S(n, k, m)\right)-2\left\lfloor\frac{n}{2}\right\rfloor \\
& \leq(n-1)+n \cdot\left(2^{n}-S(n, k, m)\right)
\end{aligned}
$$


by $w_{0}=w_{n-k+1}=1$. This completes the proofs of Theorems 2.1 and 1.8.

Acknowledgements We are indebted to the anonymous referees for their useful suggestions. The first author is partially supported by the National Research, Development and Innovation Office - NKFIH under the grant SSN117879, NK104183 and K116769. The second author is partially supported by the National Natural Science Foundation of China (No. 11671320).

Funding Open access funding provided by ELKH Alfréd Rényi Institute of Mathematics.

Open Access This article is licensed under a Creative Commons Attribution 4.0 International License, which permits use, sharing, adaptation, distribution and reproduction in any medium or format, as long as you give appropriate credit to the original author(s) and the source, provide a link to the Creative Commons licence, and indicate if changes were made. The images or other third party material in this article are included in the article's Creative Commons licence, unless indicated otherwise in a credit line to the material. If material is not included in the article's Creative Commons licence and your intended use is not permitted by statutory regulation or exceeds the permitted use, you will need to obtain permission directly from the copyright holder. To view a copy of this licence, visit http://creativecommonshorg/licenses/by/4.0/.

\section{References}

1. Burcsi, P., Nagy, D.: The method of double chains for largest families with excluded subposets. Electron. J. Graph Theory Appl. 1, 40-49 (2013)

2. DeBonis, A., Katona, G.O.H., Swanepoel, K.: Largest family without $A \cup B \subset C \cap D$. J. Combin. Theory Ser. A 111, 331-336 (2005)

3. Erdős, P.: On a lemma of littlewood and Offord. Bull. Am. Math. Soc. 51, 898-902 (1945)

4. Gerbner, D., Methuku, A., Nagy, D.T., Patkós, B., Vizer, M.: Vertex Turán problems for the oriented hypercube. arXiv:1807.06866

5. Gerbner, D., Methuku, A., Nagy, D.T., Patkós, B., Vizer, M.: Forbidding rank-preserving copies of a poset. Order 36, 611-620 (2019)

6. Griggs, J.R., Li, W.-T.: Progress on poset-free families of subsets. In: Recent Trends in Combinatorics, pp. 317-338 (2016)

7. Grósz, D., Methuku, A., Tompkins, C.: An improvement of the general bound on the largest family of subsets avoiding a subposet. Order 34, 113-125 (2017)

8. Katona, G.O.H.: Families of subsets having no subset containing another one with small difference. Nieuw Arch. Wiskunde 20(3), 54-67 (1972)

9. Katona, G.O.H., Tarján, T.G.: Extremal problems with excluded subgraphs in the $n$-cube. In: Graph Theory, pp. 84-93. Springer (1983)

10. Loehr, N.A., Michael, T.S.: The combinatorics of evenly spaced binomial coefficients. Integers 18 , Paper No. A89 (2018)

11. Martin, R.R., Methuku, A., Uzzell, A., Walker, S.: A simple proof for a forbidden subposet problem. Electron. J. Combin. 27, 1-31 (2020)

12. Methuku, A., Tompkins, C.: Exact forbidden subposet results using chain decompositions of the cycle. Electron. J. Combin. 22, 4-29 (2015)

13. Ramus, C.: Solution générale d'un problème d'analyse combinatoire. J. Reine Angew. Math. 11, 353355 (1834)

14. Sperner, E.: Ein Satzüber untermengen einer endlichen. Menge. Math. Z. 27, 544-548 (1928)

15. Tompkins, C., Wang, Y.: On an extremal problem involving a pair of forbidden posets. arXiv:1710.10760

Publisher's Note Springer Nature remains neutral with regard to jurisdictional claims in published maps and institutional affiliations. 